\title{
An efficient one-pot synthesis of polyhydroquinoline derivatives via Hantzsch condensation using a heterogeneous catalyst under solvent-free conditions
}

\author{
Muchchintala Maheswara, Vidavalur Siddaiah, Guri Lakishmi Vasantha Damu, and \\ Chunduri Venkata Rao*
}

Department of Chemistry, Sri Venkateswara University, Tirupati-517 502, India

E-mail:cvr_svu@yahoo.com

\begin{abstract}
An efficient Hantzsch condensation of polyhydroquinoline derivatives was reported via a fourcomponent coupling reaction of aldehydes, dimedone, ethyl acetoacetate and ammonium acetate in the presence of $\mathrm{HClO}_{4}-\mathrm{SiO}_{2}$ under solvent-free conditions. Operational simplicity, use of an economically convenient catalyst, high yield, short reaction times are the key features of this protocol.
\end{abstract}

Keywords: $\mathrm{HClO}_{4}-\mathrm{SiO}_{2}$, Hantzsch condensation, one-pot synthesis, polyhydroquinoline derivatives

\section{Introduction}

4-Substituted 1,4-dihydropyridines (1,4-DHPs) are well known as $\mathrm{Ca}^{2+}$ channel blockers and emerged as one of the most important classes of drugs for the treatment of cardiovascular diseases, including hypertension. ${ }^{1}$ 1,4-Dihydropyridines possess a variety of biological activities, such as, vasodilator, bronchodilator, anti-atherosclerotic, antitumor, geroprotective, hepatoprotective and antidiabetic agent. ${ }^{2 \mathrm{a}-\mathrm{d}}$ Recent studies have revealed that 1,4-DHPs exhibit several medicinal applications which include neuroprotectant ${ }^{3 \mathrm{a}}$ and platelet anti-aggregatory activity, ${ }^{3 b}$ in addition cerebral antiischemic activity in the treatment of Alzheimer's disease ${ }^{3 c}$ and as chemo sensitizer in tumor therapy. ${ }^{3 \mathrm{~d}}$ These examples clearly demonstrate the remarkable potential of novel DHP derivatives as a source of valuable drug candidates. A recent computational analysis of the comprehensive medicinal chemistry database showed the DHP framework to be among the most prolific chemo-types found. Development of drug resistance, both intrinsic drug resistance and acquired drug resistance, remains a clinical obstacle in the chemotherapy of many cancers. $^{4-5}$ Among the possible resistance modifiers, the dihydropyridines, calcium antagonists, have been studied extensively as the analogue of 
verapamil. $^{6}$ Furthermore, the photocatalytic oxidation of these compounds to pyridines has been intensively investigated. ${ }^{7}$ Relatively speaking 1,4-dihydropyridine derivatives combined with a single ring have been mostly reported. Thus, the synthesis of the heterocyclic nucleus is of continuing interest.

In view of the importance of polyhydroquinoline derivatives, many classical methods for their synthesis were reported ${ }^{8-13}$ using conventional heating and refluxing approaches in the presence of an organic solvent. These methods, however, involves long reaction times, harsh reaction conditions, the use of a large quantity of volatile organic solvents and generally leading to low yields. Therefore, it is necessary to develop an efficient and versatile method for the preparation of 1,4-dihydropyridines and the progress in this field is remarkable including recently the promotion of microwave, ${ }^{14} \mathrm{TMSCl}^{15}{ }^{15}$ ionic liquids, ${ }^{16,17}$ polymers ${ }^{18,19}$ and $\mathrm{Yb}(\mathrm{OTf})_{3} .{ }^{20}$ Moreover, to the best of our knowledge no report has been made so far about the use of $\mathrm{HClO}_{4}-\mathrm{SiO}_{2}$ as catalyst in the Hantzsch condensation. Recently $\mathrm{HClO}_{4}-\mathrm{SiO}_{2}$ has been used to catalyze the acetylation of alcohols and phenols using acetic acid. ${ }^{21}$ The multi-component reactions are powerful tools in the modern drug discovery process and allow fast, automated and high throughput generation of organic compounds. ${ }^{22}$ The possibility of performing multicomponent reactions under solvent-free conditions with a heterogeneous catalyst could enhance their efficiency from an economic as well as an ecological point of view. In recent years heterogeneous catalysts are gaining more importance due to environmental-economic factors. The catalyst is generally of low cost and can be easily handled or removed.

Herein, we would like to report a facile Hantzsch condensation in the presence of a heterogeneous catalyst under solvent-free conditions, using substituted aldehydes (1), dimedone (2), ethyl acetoacetate (3) and ammonium acetate to produce the polyhydroquinoline derivatives (4) in excellent yields.

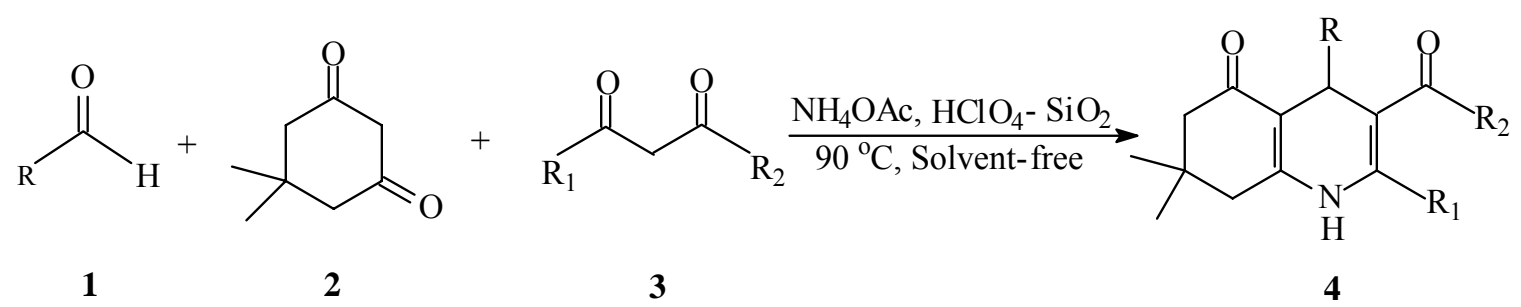

\section{Scheme 1}

\section{Results and Discussion}

The experimental procedure is very simple, convenient, and has the ability to tolerate a variety of other functional groups such as methoxy, nitro, hydroxyl, halides and olefins under the reaction conditions. Thus, we have selected the optimized reaction conditions to examine the generality of this catalyst application. Various aromatic, aliphatic, unsaturated and heterocyclic aldehydes were selected to undergo the Hantzsch condensation in the presence of this heterogeneous 
catalyst $\left(\mathrm{HClO}_{4}-\mathrm{SiO}_{2}\right)$. The results of this study are summarized in (Table-1). It was indicated that both electron rich and electron deficient aldehydes as well as heterocyclic ones such as furfural, thiophene-2-carboxaldehyde, thiophene-3-carboxaldehyde and pyridine-3carboxaldehyde worked well, mostly leading to high yields of products.

Table 1. $\mathrm{HClO}_{4}-\mathrm{SiO}_{2}$ Catalyzed Hantzsch condensation of polyhydroquinoline derivatives under solvent-free conditions

\begin{tabular}{|c|c|c|c|c|c|c|c|c|}
\hline Entry & $\mathrm{R}$ & $\mathrm{R}_{1}$ & $\mathrm{R}_{2}$ & $\begin{array}{l}\text { Time } \\
(\min )\end{array}$ & $\begin{array}{l}\text { Pro- } \\
\text { duct }\end{array}$ & $\begin{array}{c}\text { Yield } \\
(\%)\end{array}$ & $\begin{array}{l}\text { MP } \\
\left({ }^{\circ} \mathrm{C}\right) \\
\text { Found }\end{array}$ & $\begin{array}{l}\text { MP }\left({ }^{\circ} \mathrm{C}\right) \\
\text { Reported }\end{array}$ \\
\hline 1 & $\mathrm{C}_{6} \mathrm{H}_{5}$ & $\mathrm{CH}_{3}$ & OEt & 8 & $4 \mathbf{a}$ & 95 & 203-204 & $202-204^{20}$ \\
\hline 2 & $4-\mathrm{CH}_{3}-\mathrm{C}_{6} \mathrm{H}_{4}$ & $\mathrm{CH}_{3}$ & $\mathrm{OEt}$ & 12 & $4 b$ & 92 & $261-262$ & $260-261^{20}$ \\
\hline 3 & $4-\mathrm{CH}_{3} \mathrm{O}-\mathrm{C}_{6} \mathrm{H}_{4}$ & $\mathrm{CH}_{3}$ & OEt & 10 & $4 c$ & 96 & $256-257$ & $257-259^{20}$ \\
\hline 4 & $4-\mathrm{Cl}-\mathrm{C}_{6} \mathrm{H}_{4}$ & $\mathrm{CH}_{3}$ & $\mathrm{OEt}$ & 10 & $4 d$ & 95 & $245-246$ & $245-246^{16}$ \\
\hline 5 & $4-\mathrm{NO}_{2}-\mathrm{C}_{6} \mathrm{H}_{4}$ & $\mathrm{CH}_{3}$ & $\mathrm{OEt}$ & 14 & $4 e$ & 90 & $243-244$ & $242-244^{16}$ \\
\hline 6 & $2,4-\mathrm{Cl}_{2}-\mathrm{C}_{6} \mathrm{H}_{3}$ & $\mathrm{CH}_{3}$ & OEt & 15 & $4 f$ & 92 & $242-243$ & $241-243^{20}$ \\
\hline 7 & $2-\mathrm{Cl}-\mathrm{C}_{6} \mathrm{H}_{4}$ & $\mathrm{CH}_{3}$ & OEt & 13 & $4 g$ & 94 & $207-208$ & $208-210^{16}$ \\
\hline 8 & $3,4,5-\left(\mathrm{OCH}_{3}\right)_{3}-\mathrm{C}_{6} \mathrm{H}_{2}$ & $\mathrm{CH}_{3}$ & OEt & 20 & $4 h$ & 89 & $199-200$ & $198-199^{9}$ \\
\hline 9 & $\mathrm{C}_{6} \mathrm{H}_{5} \mathrm{CH}=\mathrm{CH}$ & $\mathrm{CH}_{3}$ & $\mathrm{OEt}$ & 16 & $4 i$ & 90 & $204-205$ & $204-206^{20}$ \\
\hline 10 & $2-\mathrm{NO}_{2}-\mathrm{C}_{6} \mathrm{H}_{4}$ & $\mathrm{CH}_{3}$ & OEt & 12 & $4 \mathbf{j}$ & 89 & $206-207$ & $206-208^{9}$ \\
\hline 11 & $3-\mathrm{NO}_{2}-\mathrm{C}_{6} \mathrm{H}_{4}$ & $\mathrm{CH}_{3}$ & OEt & 14 & $4 \mathbf{k}$ & 86 & $178-179$ & $177-178^{9}$ \\
\hline 12 & $4-\mathrm{F}-\mathrm{C}_{6} \mathrm{H}_{4}$ & $\mathrm{CH}_{3}$ & $\mathrm{OEt}$ & 10 & 41 & 92 & $185-186$ & $184-186^{20}$ \\
\hline 13 & $3,4,-\left(\mathrm{OCH}_{3}\right)_{2}-\mathrm{C}_{6} \mathrm{H}_{3}$ & $\mathrm{CH}_{3}$ & OEt & 20 & $4 m$ & 95 & 198-199 & - \\
\hline 14 & $4-\mathrm{N}\left(\mathrm{CH}_{3}\right)_{2}-\mathrm{C}_{6} \mathrm{H}_{4}$ & $\mathrm{CH}_{3}$ & OEt & 15 & $4 n$ & 90 & $262-263$ & $263-264^{13}$ \\
\hline 15 & 2- furyl & $\mathrm{CH}_{3}$ & OEt & 12 & 40 & 92 & $247-248$ & $246-248^{20}$ \\
\hline 16 & 2-thienyl & $\mathrm{CH}_{3}$ & $\mathrm{OEt}$ & 15 & $4 p$ & 96 & $239-240$ & $238-240^{20}$ \\
\hline 17 & 3-thienyl & $\mathrm{CH}_{3}$ & OEt & 16 & $\mathbf{4 q}$ & 91 & $246-247$ & $248-250^{16}$ \\
\hline 18 & 3-pyridyl & $\mathrm{CH}_{3}$ & $\mathrm{OEt}$ & 20 & $4 r$ & 94 & $66-67$ & $66-67^{20}$ \\
\hline 19 & $4-\mathrm{OH}-3-\mathrm{CH}_{3} \mathrm{O}-\mathrm{C}_{6} \mathrm{H}_{3}$ & $\mathrm{CH}_{3}$ & OEt & 14 & $4 s$ & 89 & $211-212$ & $210-212^{16}$ \\
\hline 20 & $4-\mathrm{OH}-\mathrm{C}_{6} \mathrm{H}_{4}$ & $\mathrm{CH}_{3}$ & OEt & 18 & $4 t$ & 90 & $230-231$ & $232-234^{20}$ \\
\hline 21 & $4-\mathrm{Br}-\mathrm{C}_{6} \mathrm{H}_{4}$ & $\mathrm{CH}_{3}$ & OEt & 16 & $4 u$ & 92 & $252-253$ & $253-255^{20}$ \\
\hline 22 & $\mathrm{C}_{2} \mathrm{H}_{5}$ & $\mathrm{CH}_{3}$ & $\mathrm{OEt}$ & 15 & $4 v$ & 83 & $145-146$ & $145-146^{20}$ \\
\hline 23 & $\mathrm{n}-\mathrm{C}_{3} \mathrm{H}_{7}$ & $\mathrm{CH}_{3}$ & OEt & 13 & $4 w$ & 81 & $146-147$ & $147-148^{20}$ \\
\hline 24 & $3,4,5-\left(\mathrm{OCH}_{3}\right)_{3}-\mathrm{C}_{6} \mathrm{H}_{2}$ & $\mathrm{CH}_{3}$ & $\mathrm{OMe}$ & 20 & $4 x$ & 87 & $220-221$ & $220-224^{9}$ \\
\hline 25 & $4-\mathrm{N}\left(\mathrm{CH}_{3}\right)_{2}-\mathrm{C}_{6} \mathrm{H}_{4}$ & $\mathrm{CH}_{3}$ & $\mathrm{OMe}$ & 16 & $4 y$ & 85 & $257-258$ & $258^{9}$ \\
\hline
\end{tabular}

The structures of the products were determined from their spectroscopic (IR, 1H NMR and MS) data. 
However, aliphatic aldehydes afforded relevant lower yields (entry 22-23). All the products were identified by comparison of analytical data (IR, NMR and MS) with those reported of authentic samples.

\section{Conclusions}

In conclusion, we have developed a simple and efficient method for the synthesis of polyhydroquinoline derivatives via Hantzsch condensation using a $\mathrm{HClO}_{4}-\mathrm{SiO}_{2}$ catalyst under solvent-free conditions. The mildness of the conversion, the experimental simplicity, the compatibility with various functional groups, the inexpensive reagents, the high yields and regioselectivity, the short reaction times and the easy workup procedure employed, makes this procedure very attractive to synthesize a variety of these derivatives.

\section{Experimental Section}

General Procedures. Melting points were determined on a Kofler hot-stage apparatus and are uncorrected. IR spectra were recorded in $\mathrm{KBr}$ discs on a Bio-Rad win FT-IR spectrophotometer. ${ }^{1} \mathrm{H}-\mathrm{NMR}$ spectra were recorded on a Varian Gemini 400 \& 200 spectrometer. LCMS was recorded on a Agilent-1100 periods LC/ MSD (VL). Elemental analyses were performed on a Vario EL-III. TLC was carried out on $\mathrm{GF}_{254}$ silica gel plates.

General experimental procedure. A mixture of aldehyde $(1 \mathrm{mmol})$, dimedone $(1 \mathrm{mmol})$, ethyl acetoacetate $(1 \mathrm{mmol})$, ammonium acetate $(1.5 \mathrm{mmol})$ and $\mathrm{HClO}_{4}-\mathrm{SiO}_{2}(50 \mathrm{mg})$ was heated at 90 ${ }^{\circ} \mathrm{C}$ with stirring for 8-20 min. and the solid product gradually formed. After completion of the reaction as indicated by TLC, the resulting solid product was treated with EtOAc followed by water and a brine solution and dried with anhydrous $\mathrm{Na}_{2} \mathrm{SO}_{4}$. The solution was concentrated in vacuum to afford the crude product. The pure product was obtained by further recrystallization using absolute alcohol. The spectral and analytical data for selected compounds are presented below.

Ethyl-1,4,7,8-tetrahydro-2,7,7-trimethyl-4-(4-fluorophenyl)-5(6H)-oxoquinolin-3-carboxylate (4l). Yellow solid, mp 185-186 ${ }^{\circ} \mathrm{C}$. IR (KBr): 3292, 2959, 1696, 1649, 1608, 1487, 1380, 1219, 1025, $764 \mathrm{~cm}^{-1} .{ }^{1} \mathrm{H}$ NMR (400 MHz, $\left.\mathrm{CDCl}_{3}\right): \delta=0.92\left(\mathrm{~s}, 3 \mathrm{H}, \mathrm{CH}_{3}\right), 1.07\left(\mathrm{~s}, 3 \mathrm{H}, \mathrm{CH}_{3}\right)$, $1.18\left(\mathrm{t}, J=7.3 \mathrm{~Hz}, 3 \mathrm{H}, \mathrm{CH}_{3}\right), 2.13-2.25\left(\mathrm{~m}, 4 \mathrm{H}, 2 \times \mathrm{CH}_{2}\right), 2.38\left(\mathrm{~s}, 3 \mathrm{H}, \mathrm{CH}_{3}\right), 4.05(\mathrm{q}, J=7.33$ $\left.\mathrm{Hz}, 2 \mathrm{H}, \mathrm{CH}_{2}\right), 5.02(\mathrm{~s}, 1 \mathrm{H}, \mathrm{CH}), 5.8(\mathrm{~s}, 1 \mathrm{H}, \mathrm{NH}), 6.85-6.89(\mathrm{~m}, 2 \mathrm{H}, \mathrm{ArH}), 7.23-7.27(\mathrm{~m}, 2 \mathrm{H}$, ArH). ${ }^{13} \mathrm{C}$ NMR $\left(75 \mathrm{MHz}, \mathrm{CDCl}_{3}\right) \delta 14.1,18.2,26.4,29.0,32.1,35.2,50.1,50.3,59.0,103.4$, $110.0,114.2,114.3,114.4,129.0,129.1,144.1,145.1,149.4,169.8,194.2$. LCMS: $\mathrm{m} / \mathrm{z}=356$ $(\mathrm{M}-\mathrm{H})^{-}$. Anal. Calcd for $\mathrm{C}_{21} \mathrm{H}_{24} \mathrm{NO}_{3} \mathrm{~F}: \mathrm{C}, 70.58 ; \mathrm{H}, 6.72 ; \mathrm{N}, 3.92 ; \mathrm{F}, 5.32$. Found: $\mathrm{C}, 70.52 ; \mathrm{H}$, $6.79 ; \mathrm{N}, 3.87 ; \mathrm{F}, 5.28$. 
Ethyl-1,4,7,8-tetrahydro-2,7,7-trimethyl-4-(3,4-dimethoxylphenyl)-5(6H)-oxoquinolin-3carboxylate (4m). Yellow solid, mp 198-199 ${ }^{\circ} \mathrm{C}$. IR (KBr): 3239, 2956, 1694, 1644, 1609, 1488, 1379, 1217, 1027, $753 \mathrm{~cm}^{-1} .{ }^{1} \mathrm{H}$ NMR: (400 MHz, $\left.\mathrm{CDCl}_{3}\right) \delta=0.95$ (s, 3H, CH ), 1.07 (s, 3H, $\left.\mathrm{CH}_{3}\right), 1.21\left(\mathrm{t}, J=7.3 \mathrm{~Hz}, 3 \mathrm{H}, \mathrm{CH}_{3}\right), 2.19-2.35\left(\mathrm{~m}, 4 \mathrm{H}, 2 \times \mathrm{CH}_{2}\right), 2.38\left(\mathrm{~s}, 3 \mathrm{H}, \mathrm{CH}_{3}\right), 3.83(\mathrm{~s}, 3 \mathrm{H}$ $\left.\mathrm{OCH}_{3}\right), 3.81\left(\mathrm{~s}, 3 \mathrm{H} \mathrm{OCH}_{3}\right) 4.07$ (q, $\left.J=7.3 \mathrm{~Hz}, 2 \mathrm{H}, \mathrm{CH}_{2}\right), 5.02(\mathrm{~s}, 1 \mathrm{H}, \mathrm{CH}), 5.92(\mathrm{~s}, 1 \mathrm{H}, \mathrm{NH})$, $6.70(\mathrm{~d}, J=8.30 \mathrm{~Hz}, 1 \mathrm{H}, \mathrm{ArH}), 6.78$ (dd, $J=8.30$ and $1.96 \mathrm{~Hz}, 1 \mathrm{H}, \mathrm{ArH}), 6.92$ (d, $J=1.96 \mathrm{~Hz}$, $1 \mathrm{H}, \mathrm{ArH}) .{ }^{13} \mathrm{C} \mathrm{NMR}\left(75 \mathrm{MHz}, \mathrm{CDCl}_{3}\right) \quad \delta 14.2,18.2,26.4,29.1,32.0,35.0,50.2,50.5,55.2$, 55.3, 59.0, 103.8, 110.1, 111.4, 111.7, 119.2, 140.4, 144.5, 146.9, 147.9, 149.4, 166.9, 194.3. LCMS: $m / z=398(\mathrm{M}-\mathrm{H})^{-}$. Anal. Calcd for $\mathrm{C}_{23} \mathrm{H}_{29} \mathrm{NO}_{5}$ : C, 69.17; H, 7.26; N, 3.50; Found: C, 68.91, H, 7.30 N, 3.46.

Ethyl-1,4,7,8-tetrahydro-2,7,7-trimethyl-4-(4-methoxylphenyl)-5(6H)-oxoquinolin-3-carboxylate (4c). Yellow solid, mp 256-257 ${ }^{\circ} \mathrm{C}$. IR (KBr): 3276, 2956, 1703, 1648, 1606, 1496, $1381,1215,1031,765 \mathrm{~cm}^{-1} .{ }^{1} \mathrm{H}$ NMR $\left(200 \mathrm{MHz}, \mathrm{CDCl}_{3}+\mathrm{DMSO}_{6}\right): \delta=0.95\left(\mathrm{~s}, 3 \mathrm{H}, \mathrm{CH}_{3}\right)$, $1.09\left(\mathrm{~s}, 3 \mathrm{H}, \mathrm{CH}_{3}\right), 1.21\left(\mathrm{t}, J=7.2 \mathrm{~Hz}, 3 \mathrm{H}, \mathrm{CH}_{3}\right), 2.01-2.10\left(\mathrm{~m}, 4 \mathrm{H}, 2 \times \mathrm{CH}_{2}\right), 2.30\left(\mathrm{~s}, 3 \mathrm{H}, \mathrm{CH}_{3}\right)$, $3.70\left(\mathrm{~s}, 3 \mathrm{H} \mathrm{OCH}_{3}\right), 4.00\left(\mathrm{q}, J=7.2 \mathrm{~Hz}, 2 \mathrm{H}, \mathrm{CH}_{2}\right), 4.80(\mathrm{~s}, 1 \mathrm{H}, \mathrm{CH}), 6.65$ (d, J = $7.3 \mathrm{~Hz}, 2 \mathrm{H}$, $\operatorname{ArH}), 7.10$ (d, J=7.3 Hz, 2H, ArH ), 8.65 (s, 1H, NH). ${ }^{13} \mathrm{C}$ NMR (75 MHz, DMSO-d 6 ) $\delta 14.1$, 18.2, 26.4, 29.1, 32.1, 34.7, 50.2, 50.5, 54.8, 58.9, 103.2, 110.1, 113.0, 113.1, 128.2, 128.3, 139.8, 144.6, 149.1, 157.2, 166.9, 194.2. LCMS: $\mathrm{m} / \mathrm{z}=368(\mathrm{M}-\mathrm{H})^{-}$. Anal. Calcd for $\mathrm{C}_{22} \mathrm{H}_{27} \mathrm{NO}_{4}$ : C, 71.54; H, 7.31; N, 3.79; Found: C, 71.59; H, 7.35; N, 3.84.

Ethyl-1,4,7,8-tetrahydro-2,7,7-trimethyl-4-(4-hydroxy-3-methoxylphenyl)-5(6H)-

oxoquinolin-3-carboxylate (4s). Yellow solid, mp 211-212 ${ }^{\circ} \mathrm{C}$. IR (KBr): 3388, 2953, 1700, 1643, 1589, 1482, 1385, 1218, 1029, $782 \mathrm{~cm}^{-1}$. ${ }^{1} \mathrm{H}$ NMR $\left(200 \mathrm{MHz}, \mathrm{CDCl}_{3}+\mathrm{DMSO}_{6}\right): \delta=$ $0.95\left(\mathrm{~s}, 3 \mathrm{H}, \mathrm{CH}_{3}\right), 1.09\left(\mathrm{~s}, 3 \mathrm{H}, \mathrm{CH}_{3}\right), 1.25\left(\mathrm{t}, J=7.2 \mathrm{~Hz}, 3 \mathrm{H}, \mathrm{CH}_{3}\right), 2.02-2.20\left(\mathrm{~m}, 4 \mathrm{H}, 2 \times \mathrm{CH}_{2}\right)$, $2.30\left(\mathrm{~s}, 3 \mathrm{H}, \mathrm{CH}_{3}\right), 3.80(\mathrm{~s}, 3 \mathrm{H} \mathrm{OCH})_{3}, 4.05\left(\mathrm{q}, J=7.2 \mathrm{~Hz}, 2 \mathrm{H}, \mathrm{CH}_{2}\right), 4.80(\mathrm{~s}, 1 \mathrm{H}, \mathrm{CH}), 6.60$ (s $2 \mathrm{H}, \mathrm{ArH}), 6.82(\mathrm{~s}, 1 \mathrm{H}, \mathrm{ArH}), 7.69(\mathrm{~s}, \mathrm{OH}), 8.49(\mathrm{~s}, 1 \mathrm{H}, \mathrm{NH}) .{ }^{13} \mathrm{C} \mathrm{NMR}\left(75 \mathrm{MHz}, \mathrm{DMSO}-\mathrm{d}_{6}\right) \delta$ $14.2,18.2,26.3,29.2,32.1,35.0,50.2$, 55.4, 58.9, 104.0, 110.1, 112.0, 114.9, 119.3, 119.5, 139.0, 144.3, 144.5, 146.7, 149.2, 167.0, 194.3. LCMS: $m / z=384(\mathrm{M}-\mathrm{H})^{-}$. Anal. Calcd for $\mathrm{C}_{22} \mathrm{H}_{27} \mathrm{NO}_{5}$ : C, 68.57; H, 7.06; N, 3.64; Found: C, 68.52; H, 7.10; N, 3.60

Ethyl-1,4,7,8-tetrahydro-2,7,7-trimethyl-4-(2-chlorolphenyl)-5(6H)-oxoquinolin-3-carboxylate (4g). Yellow solid, mp 207-208 ${ }^{\circ} \mathrm{C}$. IR (KBr): 3063, 2956, 1721, 1640, 1611, 1467, 1384, 1227, 1021, $745 \mathrm{~cm}^{-1}$. ${ }^{1} \mathrm{H}$ NMR (200 MHz, $\left.\mathrm{CDCl}_{3}+\mathrm{DMSO}_{6}\right): \delta=0.95\left(\mathrm{~s}, 3 \mathrm{H}, \mathrm{CH}_{3}\right), 1.05(\mathrm{~s}$, $\left.3 \mathrm{H}, \mathrm{CH}_{3}\right), 1.20\left(\mathrm{t}, J=7.2 \mathrm{~Hz}, 3 \mathrm{H}, \mathrm{CH}_{3}\right), 2.01-2.21\left(\mathrm{~m}, 4 \mathrm{H}, 2 \times \mathrm{CH}_{2}\right), 2.40\left(\mathrm{~s}, 3 \mathrm{H}, \mathrm{CH}_{3}\right), 4.05$ (q, $\left.J=7.2 \mathrm{~Hz}, 2 \mathrm{H}, \mathrm{CH}_{2}\right), 4.60(\mathrm{~s}, 1 \mathrm{H}, \mathrm{CH}), 7.10-7.30(\mathrm{~m} \mathrm{4H}, \mathrm{ArH}), 7.60(\mathrm{~s}, 1 \mathrm{H}, \mathrm{NH}) .{ }^{13} \mathrm{C} \mathrm{NMR}(75$ MHz, DMSO-d 6 ) $\delta$ 26.5, 29.1, 31.2, 32.3, 42.4, 46.8, 48.9, 50.5, 52.9, 55.1, 101.3, 109.2, 114.2, 125.9, 127.1, 128.7, 131.1, 132.3, 141.0, 167.5, 195.7. LCMS: $m / z=372(\mathrm{M}-\mathrm{H})^{-} . \mathrm{C}_{21} \mathrm{H}_{24} \mathrm{NO}_{3} \mathrm{Cl}$ : Anal. Calcd for C, 67.56; H, 6.43; N, 3.75; Cl, 9.38; Found: C, 67.52; H, 6.48; N, 3.72; Cl, 9.42 


\section{References}

1. (a) Bossert, F.; Meyer, H.; Wehinger, E. Angew. Chem., Int. Ed. Engl. 1981, 20, 762. (b) Nakayama, H.; Kasoaka, Y. Heterocycles 1996, 42, 901.

2. (a) Godfraid, T.; Miller, R.; Wibo, M. Pharmocol. Rev. 1986, 38, 321. (b) Sausins, A.; Duburs, G. Heterocycles 1988, 27, 269. (c) Mager, P. P.; Coburn, R. A.; Solo, A. J.; Triggle, D. J.; Rothe, H. Drug Design Discovery 1992, 8, 273. (d) Mannhold, R.; Jablonka, B.; Voigdt, W.; Schoenafinger, K.; Schravan, K. Eur. J. Med. Chem. 1992, 27, 229.

3. (a) Klusa, V. Drugs Fut. 1995, 20, 135. (b) Bretzel, R. G.; Bollen, C. C.; Maeser, E.; Federlin, K. F. Am. J. Kidney. Dis. 1993, 21, 53. (c) Bretzel, R. G.; Bollen, C. C.; Maeser, E.; Federlin, K. F. Drugs Fut. 1992, 17, 465. (d) Boer, R.; Gekeler, V. Drugs Fut. 1995, 20, 499.

4. Davis, H. L.; Davis, T. E. Cancer Treat. Rep. 1979, 63, 809.

5. Pastan, I.; Gottesman, M. M. N. Engl. J. Med. 1987, 316, 1388.

6. Tanabe, H.; Tasaka, S.; Ohmori, H.; Gomi, N.; Sasaki, Y.; Machida, T.; Lino, M.; Kiue, A.; Naito, S.; Kuwano, M. Bioorg. Med. Chem. 1998, 6, 2219.

7. Zhang, D.; Wu, L. Z.; Zhou, L.; Han, X.; Yang, Q. Z.; Zhang, L. P.; Tung, C. H. J. Am. Chem. Soc. 2004, 126, 3440.

8. Hantzsch, A. Ann. Chem. 1882, I, 215.

9. Sainani, J. B.; Shah, A. C.; Arya, V. P. Indian J. Chem, Sect B 1994, 33, 526.

10. Ahluwalia, V. K.; Goyal, B.; Das, U. J. Chem. Res., Synop. 1997, 266.

11. Margarita, S.; Estael, O.; Yamila, V.; Beatriz, P.; Lourdes, M.; Nazario, M.; Margarita. Q.; Carlos, S.; Jose, L. S.; Hector, N.; Norbert, B.; Oswald, M. P. Tetrahedron 1999, 55, 875.

12. Ahluwalia, V. K.; Goyal, B. Das, U. J. Chem. Res., Miniprint. 1997, 7, 1501.

13. Ahluwalia, V. K.; Goyal, B. Indian J. Chem, Sect. B 1996. 35, 1021.

14. Tu, S.-J.; Zhou, J.-F.; Deng, X.; Cai, P.-J.; Wang, H.; Feng, J.-C. Chin. J. Org. Chem. 2001, $21,313$.

15. Sabitha, G.; Reddy, G. S. K. K.; Reddy, C. S.; Yadav, J. S. Tetrahedron Lett. 2003, 44, 4129.

16. Ji, S. J.; Jiang, Z. Q.; Lu, J.; Loa, T. P. Synlett 2004, 831.

17. Sridhar, R.; Perumal, P. T. Tetrahedron 2005, 61, 2465.

18. Breitenbucher, J. G.; Figliozzi, G. Tetrahedron Lett. 2000, 41, 4311.

19. Dondoni, A.; Massi, A.; Minghini, E.; Bertolasi, V. Tetrahedron 2004, 60, 2311.

20. Wang, L. M.; Sheng, J.; Zhang, L.; Han, J. W.; Fan, Z. Y.; Tian, H.; Qian, C. T. Tetrahedron 2005, 61, 1539.

21. Chakraborti, A. K.; Gulhane, R. Chem. Comm. 2003, 1896.

22. Weber, L. Curr. Med. Chem. 2002, 9, 1241. 